\title{
Rate-Dependent Effect of Amphetamine in Rats: Extension to Between-Subjects Effect
}

\author{
MICHAEL D. BEECHER*** and DONALD E. JACKSON \\ Department of Psychology, Eastern Michigan University, Ypsilanti, Michigan 48197, U.S.A.
}

Received February 25, 1975; Final Version January 15, 1976

\begin{abstract}
Following either variable-interval or fixed-interval training, 20 rats received both 0.5 and $2.0 \mathrm{mg} / \mathrm{kg}$ of amphetamine. For both schedules, amphetamine decreased response rates of high-rate subjects and increased those of low-rate
\end{abstract}

subjects. Within-subject analysis of fixed-interval rates revealed the same rate-dependent effect. It is suggested that the between-subject and within-subject effects may have the - same basis.

Key words: Amphetamine - Rate dependency - Operant behavior.

The effect of amphetamine on operant behavior of individual subjects depends on the subject's baseline (control) response rate. It is generally found that the rate-increasing effect of the drug decreases as the baseline rate increases, until a certain rate is reached at which no effect occurs. Beyond this "neutral point", a rate-decreasing effect occurs, which increases as the baseline rate increases further. When the drug response rate is expressed as a percentage of the control response rate ("output ratio") and plotted as a function of the control rate on log-log coordinates, the resulting function is linear, with the $\mathrm{x}$-intercept marking the transition between rate-increasing and rate-decreasing effects. The slope and intercepts, in turn, vary with the drug dose. A review of these "ratedependency" and "dose-dependency" effects can be found in Kelleher and Morse (1968).

The rate-dependency effect has been demonstrated by manipulating the subject's response rate via different schedules of reinforcement. For example, differential-reinforcement-of-low-rate (DRL) and fixed-ratio (FR) schedules have been used to engender low and high rates of responding, respectively (e.g., Kelleher et al., 1961): amphetamine increases the DRLproduced low rate and decreases the FR-produced high rate. A fixed-interval $(F I)$ schedule produces a variety of rates within each interval, the lowest rate occurring at the beginning of the interval and the highest rate occurring just before reinforcement. It has been found that amphetamine increases the low rates occurring early in the interval, while decreasing

\footnotetext{
* Also Kresge Hearing Research Institute, University of Michigan.

** To whom offprint requests should be sent.
}

the high terminal rates (Smith, 1964; Kelleher and Morse, 1968; McMillan, 1969, 1973).

It has not yet been determined whether the ratedependency generalization applies between subjects: that is, for a given schedule, will amphetamine increase the rates of low-rate responding subjects, while decreasing the rates of high-rate subjects, and will these changes be describable by a simple regression line. Such an extension would widen the scope of the rate-dependency generalization. If the baseline response rate itself is the primary determinant of the drug effect (for a given schedule at a given dose), then it is to be expected that a between-subjects function (different rate values generated by different subjects on the same schedule) would be similar to withinsubjects functions (different rate values generated by one subject on several different schedules, or different segments of a schedule). If, on the other hand, different variables underlie between-subject and within-subject (schedule-induced) rate differences, then it is possible that a between-subject rate-dependency effect would be poorly described by a linear function, or perhaps would not be found at all.

There is little direct evidence bearing on the similarity of between-subject and within-subject ratedependency functions in the animal literature, since these studies have generally utilized within-subject designs. Several studies, aimed at different problems, have incidentally used a between-subject design which allows comparison of subjects with different characteristic baseline rates (Green and Harvey, 1974; Hearst, 1961; Ray and Bivens, 1966). These studies have all failed to reveal a between-subject ratedependency. None of them, however, was specifically designed to examine this phenomenon and the failure 
may be attributable to the low range of baseline response rates generated (approximately $2: 1$ in Green and Harvey, 1974, and Ray and Bivens, 1966; 4:1 in Hearst, 1961). Recently Heffner et al. (1974) reported that rats with extremely high or extremely low baseline rates on FR 20 and FI 2 showed rate changes consistent with the rate-dependency interpretation. They say, however, that in general there was no consistent correlation between individual baseline response rates and drug effect.

The purpose of the present study was to examine the rate-dependent effect of amphetamine where the different rates were generated between subjects. One group of ten rats was trained on a variable-interval (VI) schedule. This schedule was chosen since it engenders a constant rate within a given subject, yet individual rats will vary considerably in their characteristic rates on the schedule. A second group of ten subjects was trained on a fixed-interval (FI) schedule. Use of this schedule allowed an additional examination of within-subjects rate-dependency functions.

\section{Method}

Subjects. Twenty male rats (Sprague-Dawley strain) were maintained at $85 \%$ of their normal body weight. The mean of their $85 \%$ weights was $255 \mathrm{~g}$.

Apparatus. Ten standard operant conditioning units (Lehigh Valley Co.) were individually housed in sound- and lightresistant chests. The front wall of the unit contained a response lever and a food cup to which $45-\mathrm{mg}$ Noyes pellets could be delivered. Masking noise was provided by an exhaust fan, and illumination was from an overhead $7.5 \mathrm{w}$ bulb. Experimental contingencies were programmed by solid state equipment.

Procedure. Following preliminary training rats were given seven 90 -min sessions of FI $1.5 \mathrm{~min}$ (10 rats) or VI $1.5 \mathrm{~min}$ (10 rats). On the FI schedule the first response after $1.5 \mathrm{~min}$ was reinforced with a food pellet. On the VI schedule the first response after an average of $1.5 \mathrm{~min}$ was reinforced.

Testing took place over 4 days (sessions $8-11$ ). On the first day half of each group received $0.5 \mathrm{mg} / \mathrm{kg}$ and half $2.0 \mathrm{mg} / \mathrm{kg} d$-amphetamine sulfate. Following two control days, the subjects received the drug at the reverse dosage level on the fourth day.

Data Analysis. For each animal, the response rate (responses/ min) obtained during each drug day was expressed as a percentage of the mean response rate obtained during the two intervening non-drug control days. Logarithms of these "output ratios" were plotted as a function of the logarithms of the control rates. Regression lines were fitted to the data points by the method of least squares.

For the within-subjects analysis of the FI data, responses were cumulated during each third (trimester) of the interval. These data were converted to output ratios and analyzed as described above for the between-subjects data.

Drug. $d$-Amphetamine sulfate was dissolved in $0.9 \%$ sodium chloride solution to a concentration of $0.6 \mathrm{mg} / \mathrm{ml}$ (expressed in terms of the salt). Dosage levels of 0.5 and $2.0 \mathrm{mg} / \mathrm{kg}$ (injection volumes 0.4 and $1.2 \mathrm{ml} / \mathrm{kg}$ ) were used. Drugs were injected i.p. $20 \mathrm{~min}$ prior to the experimental session.

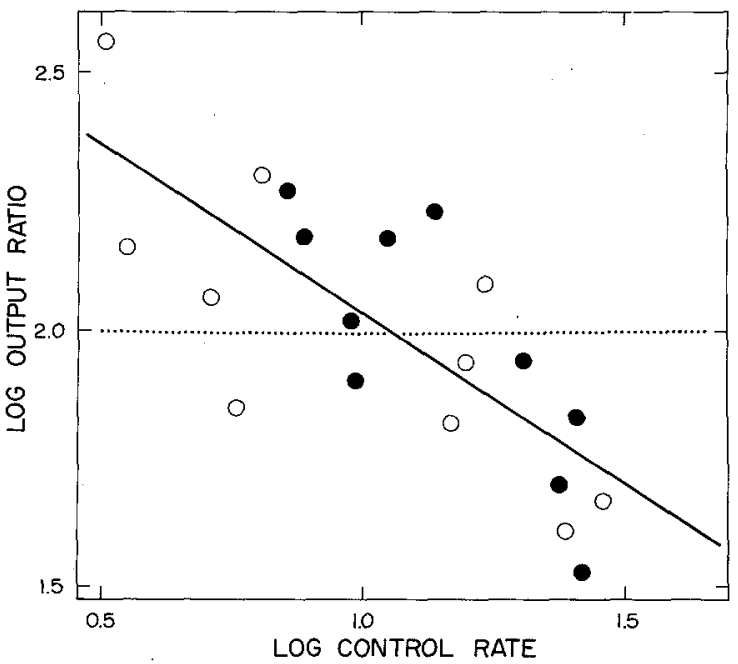

Fig. 1. Log output ratio as a function of log control rate (responses/min). Open circles represent data from 10 rats on FI schedule, filled circles 10 rats on VI. $y=0.67 x+2.70$ $(r=0.76)$

\section{Results}

Between-Subjects Analysis. Figure 1 shows log output ratios plotted as a function of log control responses/ $\mathrm{min}$ for both VI and FI groups at the $2.0 \mathrm{mg} / \mathrm{kg}$ dosage level. The FI rates are of course overall rates and are not representative of any particular time segment within a given interval (the within-interval analysis is given in the next section). The negative slope of the regression line indicates a rate-dependent effect of amphetamine: the higher the baseline rate, the less rate-increasing or more rate-decreasing was the effect of the drug. A "neutral point", where the regression line intersects the $\log$ output ratio of 2 (output ratio $=100$ ), occurs at a $\log$ control rate of 1.05 (11.2 responses/min).

The regression line of Fig. 1 fits the data well enough to account for $58 \%$ of the variance $(r=0.76$, $P<0.001)$. Separate regression lines for the FI and VI groups are not shown in Fig. 1 since they were similar to one another and to the overall regression line: slope, "neutral point" and y-intercept for the FI data were $0.63,1.00$ and 2.63 respectively $(r=0.78$, $P<0.01$ ), and for the VI data, 0.89, 1.12 and 3.00 $(r=0.79, P<0.01)$.

The overall effect of the drug at the $0.5 \mathrm{mg} / \mathrm{kg}$ dosage was much weaker, and a weaker rate-dependency effect was found. Compared to the higher dosage level, the slopes of the regression lines were less negative (VI 0.39 vs. 0.89 , FI 0.22 vs. 0.63 ) and the control rate accounted for less of the variance (VI $r=0.65, P<0.05$, FI $r=0.47, P<0.10$ ). Since the regression line slopes were not significant at the 0.01 level, these data were not analyzed further. 
Table 1. Control response rates and output ratios $(2 \mathrm{mg} / \mathrm{kg}$ ) by FI trimester, and slopes (b) and y-intercepts (a) of regression lines

\begin{tabular}{|c|c|c|c|c|c|c|c|c|}
\hline \multirow[t]{2}{*}{ Rat } & \multicolumn{3}{|c|}{ Control rate } & \multicolumn{3}{|c|}{ Output ratio } & \multirow[t]{2}{*}{$b^{a}$} & \multirow[t]{2}{*}{$\mathrm{a}^{\mathrm{a}}$} \\
\hline & I & II & III & I & II & III & & \\
\hline 1 & 0.36 & 2.76 & 12.33 & 403 & 140 & 95 & 0.31 & 2.35 \\
\hline 5 & 0.44 & 1.92 & 10.20 & 191 & 225 & 77 & 0.31 & 2.28 \\
\hline 6 & 11.70 & 20.16 & 50.30 & 67 & 58 & 44 & 0.35 & 2.26 \\
\hline 9 & 3.22 & 6.76 & 38.79 & 99 & 116 & 47 & 0.36 & 2.26 \\
\hline 10 & 1.04 & 2.18 & 6.50 & 464 & 239 & 152 & 0.71 & 2.68 \\
\hline 11 & 6.14 & 25.80 & 30.41 & 194 & 50 & 46 & 0.86 & 2.94 \\
\hline 15 & 4.42 & 16.04 & 32.26 & 443 & 156 & 109 & 0.84 & 3.23 \\
\hline 16 & 1.14 & 10.56 & 46.07 & 997 & 215 & 70 & 0.74 & 3.06 \\
\hline 19 & 1.36 & 2.64 & 6.30 & 936 & 407 & 183 & 1.09 & 3.10 \\
\hline 20 & 0.42 & 1.72 & 15.19 & 2164 & 493 & 122 & 0.78 & 2.97 \\
\hline
\end{tabular}

a Slopes are negative, intercepts are in log output ratio.

Within-Subjects Analysis. Table 1 shows control rates, output ratios, and the slopes and y-intercepts of the best-fit regression lines for each subject (not evaluated statistically since there are only three data points per subject). Only the $2.0 \mathrm{mg} / \mathrm{kg}$ data are analyzed here since the effect of the drug at $0.5 \mathrm{mg} / \mathrm{kg}$ was marginal. On the control days all FI subjects showed the typical accelerated ("scalloped") response rates over the fixed interval: for every animal, rates were lowest in trimester I, intermediate in trimester II and highest in trimester III. Output ratios for all 10 animals were lower in the third trimester than in the first or the second ( $P=0.001$ for each of the two comparisons). Thus, for both the between-subjects and within-subjects analyses, the effect of amphetamine was ratedependent, and both rate increases and rate decreases occurred.

\section{Discussion}

The major finding of this study is that the ratedependent effect of amphetamine applies between subjects as well as within subjects. The rate-increasing effect of the drug is inversely proportional to the control rate upon which it acts, with the effect becoming rate-decreasing beyond some "neutral" point.

Three conclusions seem warranted. (1) The best predictor of the effect of a given dose of amphetamine on operant responding is the control response rate. (2) Since similar predictions concerning ratedependency are made, whether between subjects or within a given subject, it seems reasonable to hypothesize that the same mechanism may be responsible for both between- and within-subjects effects. (3) Since the variable of control rate accounted for only $58 \%$ of the variance in output ratios, it is possible that certain characteristics that vary among individual subjects are also determinants of the effect of the drug independently of their effect on the subject's response rate. That is, though control rate is the best predictor of the drug effect, it is by no means the sole determinant.

\section{References}

Green, T. K., Harvey, J. A.: Enhancement of amphetamine action after interruption of ascending serotonergic pathways. J. Pharmacol. exp. Ther. 190, 109-117 (1974)

Hearst, E.: Effects of $d$-amphetamine on behavior reinforced by food and water. Psychol. Rep. 8, 301-309 (1961)

Heffner, T. G., Drawbaugh, R. B., Zigmond, M. J. : Amphetamine and operant behavior in rats: Relationship between drug effect and control response rate. J. comp. physiol. Psychol. 86, 1031-1043 (1974)

Kelleher, R. T., Fry, W., Deegan, J., Cook, L.: Effects of meprobamate on operant behavior in rats. J. Pharmacol. exp. Ther. 133, 271-280 (1961)

Kelleher, R. T., Morse, W. H.: Determinants of the specificity of behavioral effects of drugs. Ergebn. Physiol. 60, $1-56(1968)$

McMillan, D. E.: Effects of $d$-amphetamine on performance under several parameters of multiple fixed-ratio, fixedinterval schedules. J. Pharmacol. exp. Ther. 167, 26-33 (1969)

McMillan, D. E.: Drugs and punished responding. I. Ratedependent effects under multiple schedules. J. exp. Anal. Behav. 19, 133-145 (1973)

Ray, O. S., Bivens, L. W.: Chlorpromazine and amphetamine effects on three operant and on four discrete trial reinforcement schedules. Psychopharmacologia (Berl.) 10, 32-43 (1966)

Smith, C. B.: Effects of $d$-amphetamine upon operant behavior of pigeons: enhancement by reserpine. J. Pharmacol. exp. Ther. 146, 167-174 (1964) 